\title{
Systematic Uncertainties of the NOvA Neutrino Oscillation Analysis
}

\section{Tomas Nosek, for the NOvA Collaboration}

Charles University, Institute of Particle and Nuclear Physics

V Holesovickach 2, Prague, Czech Republic

E-mail: tomas.nosek@mff.cuni.cz

NOvA is a two detector long-baseline neutrino oscillation experiment using Fermilab's $700 \mathrm{~kW}$ NuMI muon neutrino beam. It studies the disappearance of muon (anti)neutrinos and the appearance of electron (anti)neutrinos in the beam over a distance of $810 \mathrm{~km}$ between the detectors. The observations can be used to constrain neutrino oscillation parameters: $\theta_{23}, \Delta m_{32}^{2}$ and $\delta_{C P}$ in the standard 3 active neutrinos paradigm. Although statistical uncertainties currently dominate in these measurements, understanding the major sources of systematic uncertainties and their correlations is vital for both the interpretation and precision of the results and also for further improvements of the analysis. This note presents details of the systematic uncertainties affecting NOvA's neutrino oscillation analysis results and how we evaluate them by generating systematically shifted predictions.

Acknowledgments: This work is supported by MSMT CR.

40th International Conference on High Energy physics - ICHEP2020

July 28 - August 6, 2020

Prague, Czech Republic (virtual meeting) 


\section{Introduction}

NOvA uses two functionally indentical detectors, near (ND) and far (FD), to observe $v_{\mu}$ disappearance and $v_{e}$ appearance in Fermilab's $700 \mathrm{~kW} \mathrm{NuMI} v_{\mu}$ beam. It has recently reported updated results on neutrino oscillations with additional $v$-beam data with a total exposure of 13.6 and $12.5 \times 10^{20}$ POT in NuMI $v$ and $\bar{v}$ modes ( 1/2 of all expected data) [1]. There were 211/105 $v_{\mu} / \bar{v}_{\mu}$ and 82/33 $v_{e} / \bar{v}_{e}$ candidates observed. The best fit estimates of oscillation parameters are $\sin ^{2} \theta_{23}=0.57_{-0.04}^{+0.03}, \Delta m_{32}^{2}=(2.41 \pm 0.07) \times 10^{-3} \mathrm{eV}^{2}, \delta_{\mathrm{CP}}=0.82 \pi$ and they correspond to the normal ordering of the neutrino masses. Below, systematic uncertainties of NOvA's 2020 neutrino oscillation measurements are discussed in brief.

\section{Sources of considered systematic uncertainties}

There are over 80 individual uncertainties considered for NOvA's oscillation analysis grouped into 7 categories (see Figs. 1 and 2). Among those with the largest impact on the total neutrino events predictions are detector calibration and neutrino cross section uncertainties. Also, the observed data/MC disagreement in the low energy neutron clusters has motivated a specific "neutron uncertainty". It is significant for energy reconstruction of $\bar{v}_{\mu}$ events (approx. 1\%). Further categories list detector response (i.e. light yields), beam flux (neutrino parent hadron production in the NuMI target), lepton reconstruction (primary lepton angle, $\mu$ stopping power) and uncertainties uncorrelated between the detectors (detector mass and acceptance, POT counting etc.).

\section{Reducing detector correlated uncertainties}

For each considered uncertainty, nominal and systematically shifted far detector (FD) predictions are generated using near detector (ND) data corrected MC. This extrapolation FD/ND technique (see e.g. Refs. [2,3] for details) is simplistically expressed bin-by-bin as

$$
\text { FD prediction }=\frac{\text { ND data corrected } \mathrm{MC}}{\mathrm{ND} \text { uncorrected } \mathrm{MC}} \times \mathrm{FD} \mathrm{MC} \text {. }
$$

It significantly cancels detector correlated uncertainties (Fig. 1), especially in $v_{e}$ samples.

Additionally, beam flux and some of the neutrino cross section uncertainties are treated with principal component analysis (PCA). PCA uses eigenvalue decomposition of a covariance matrix from an ensemble of randomly generated shifted predictions in energy bins of ND and FD/ND bases. Identifying the largest principal components helps to account for possible bin-bin correlations and reduces the number of systematic nuisance parameters included in the following fit (from tens to units) and thus reduces computation time.

\section{Uncertainties in oscillation analysis}

Uncertainties are determined for all analysis samples $\left(v_{\mu} / \bar{v}_{\mu}, v_{e} / \bar{v}_{e}\right)$ separately. Though $v_{e}$ uncertainties are (and will be) governed by the low statistics, statistical and systematic uncertainties are already comparable in the case of $v_{\mu}$ samples. For a more comprehensive insight, the effect of individual systematic categories on the neutrino oscillation parameters estimates is simulated (see 


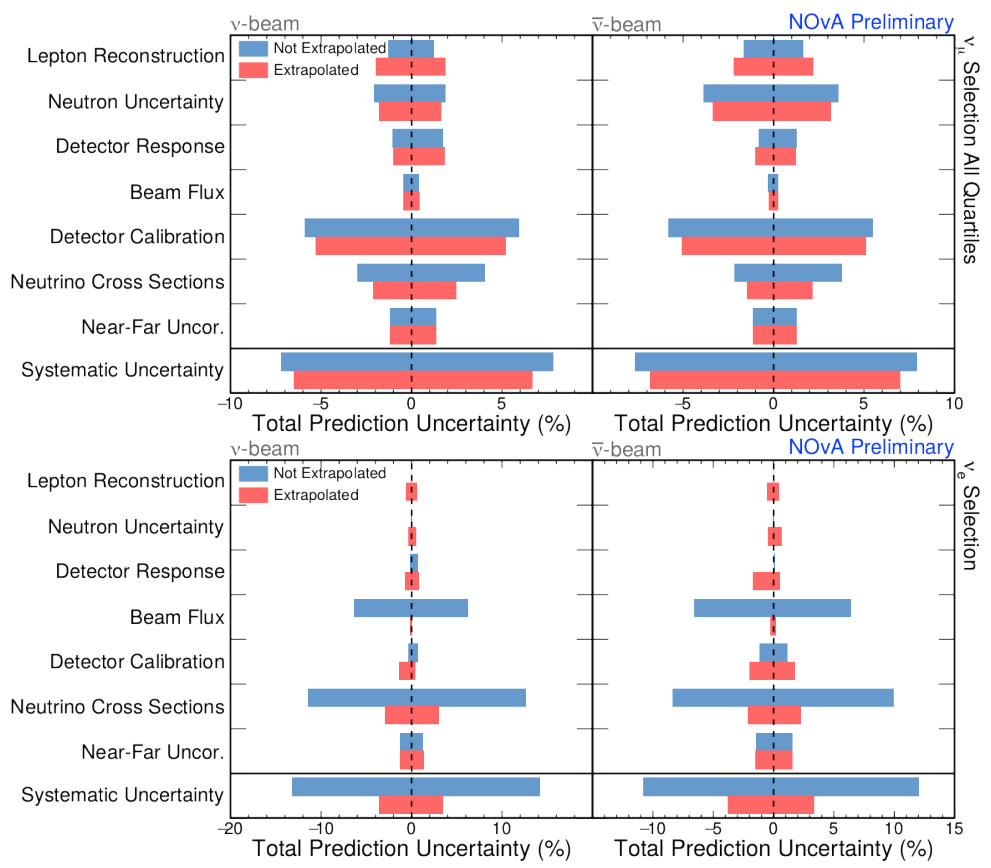

Figure 1: Comparison of estimated systematic uncertainties for total predictions of $v_{\mu}$ (top) and $v_{e}$ (bottom) candidates in neutrino (left) and antineutrino (right) NuMI beam modes when using FD/ND extrapolation technique (red, "Extrapolated") or not (blue, "Not Extrapolated").

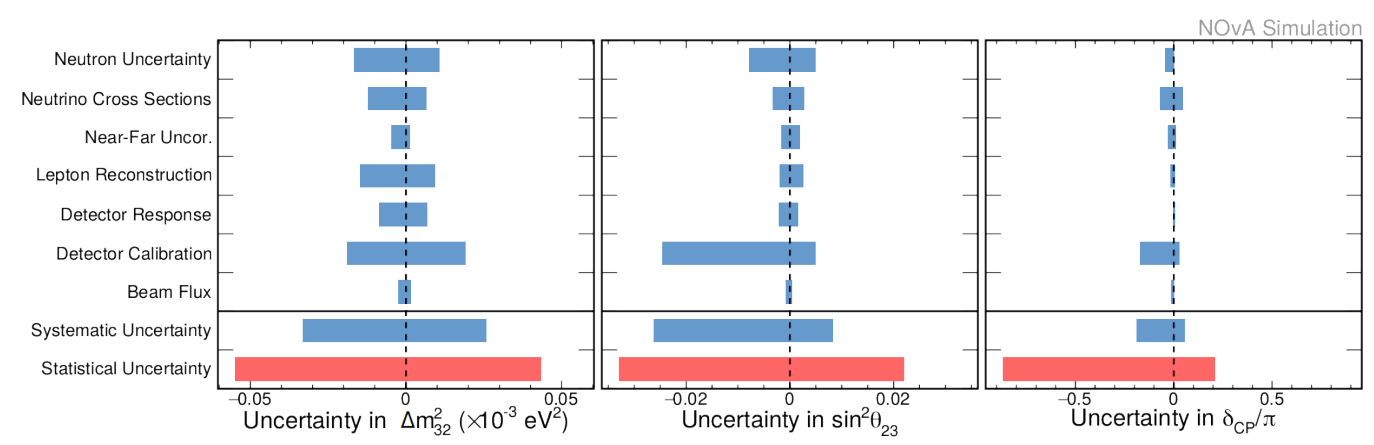

Figure 2: Uncertainties on the neutrino oscillation parameters estimates and the effect of the individual systematic categories simulated at the NOvA's best fit point.

Fig. 2). Precision on $\delta_{\mathrm{CP}}$ is limited by $v_{e}$ statistics and potential degeneracies of the parametric space: normal vs inverted mass ordering and $\sin ^{2} \theta_{23} \lessgtr 0.5$. Further reduction of calibration uncertainties (e.g. by the NOvA Test Beam Program studies [4]) would be helpful for more precise determination of $\sin ^{2} \theta_{23}$ and $\Delta m_{32}^{2}$.

\section{References}

[1] A. Himmel [NOvA], 10.5281/zenodo.4142045, July 2020

[2] L. Suter [NOvA], arXiv:1511.00181 [hep-ex]

[3] J. Wolcott [NOvA], PoS NuFACT2018 (2019) 098, 10.22323/1.341.0098

[4] A. Sutton [NOvA], PoS NuFACT2018 (2018) 058, 10.22323/1.341.0058 